\title{
Doppler tecidual do miocárdio de gatos submetidos à anestesia dissociativa
}

\author{
Tissue Doppler imaging of the myocardium in cats submitted to dissociative anesthesia
}

\author{
Daniel Cometti Borlini ${ }^{\mathrm{I} *}$ Daniel Capucho de Oliveira' ${ }^{\mathrm{I}}$ Warley Gomes dos Santos ${ }^{\mathrm{I}}$ \\ Jessica Nascimento Moraes Monteiro ${ }^{\mathrm{I}}$ Lorena Adão Vescovi ${ }^{\mathrm{II}}$ \\ Séfora Vieira da Silva Gouvêa de Barros ${ }^{\text {II }}$ Tessa Roesler ${ }^{I I}$ \\ Aloir Queiroz de Araújo Sobrinho ${ }^{\text {III }}$ \\ Fabiano Séllos Costa ${ }^{\mathrm{I}}$
}

\section{RESUMO}

O Doppler tecidual é uma técnica recente que permite avaliar a velocidade de movimentação do miocárdio durante as fases do ciclo cardíaco, fornecendo informações importantes da função cardíaca. Na medicina veterinária, estudos têm sido realizados para a caracterização de disfunções da contratilidade cardíaca e o estabelecimento de padrões de normalidade. O presente trabalho objetivou avaliar a velocidade de contração do miocárdio pela técnica de Doppler tecidual em gatos submetidos à anestesia dissociativa. Para tanto, foram utilizados nove gatos adultos, hígidos, sem distinção de sexo ou raça. Foram medidos os valores de Ea (velocidade máxima longitudinal no início da diástole), Aa (velocidade máxima longitudinal na sístole atrial) e Sa (velocidade máxima longitudinal na sístole ventricular) nas bordas lateral e septal do anel mitral. Os valores médios observados para Ea septal e Sa septal foram de $7,2 \pm 2,5 \mathrm{~cm} \mathrm{~s}^{-1}$ e $6,3 \pm 1,7 \mathrm{~cm} \mathrm{~s}^{-1}$, respectivamente. O valor médio obtido para Ea lateral foi de $12,0 \pm 4,1 \mathrm{~cm} \mathrm{~s}^{-1}$, enquanto que o valor médio de Sa lateral foi de $8,3 \pm 1,6 \mathrm{~cm} \mathrm{~s}^{-1}$. Foi obtido um valor médio de $7,0 \pm 3,1 \mathrm{~cm} \mathrm{~s}^{-1}$ para Aa lateral e $4,7 \pm 1,5 \mathrm{~cm} \mathrm{~s}^{-1}$ para Aa septal. A padronização dos valores médios da velocidade de contração do miocárdio na espécie felina é de grande importância, podendo auxiliar no diagnóstico precoce de alterações da função contrátil do coração. Em razão da escassez de estudos com a técnica de Doppler tecidual em gatos, os valores obtidos neste trabalho permitem a realização de estudos comparativos, auxiliando a aplicação dessa técnica em protocolos experimentais e no diagnóstico precoce de cardiopatias na espécie felina.

Palavras-chave: felinos, cardiologia, função diastólica.

\begin{abstract}
The tissue Doppler imaging (TDI) is a recent technique that allows the evaluation of the myocardium movement velocity during the cardiac cycle phases, providing important informations of the cardiac function. In veterinary medicine, studies have been realized for the characterization of cardiac contractility dysfunctions and to establish the normality patterns. The present work meant to evaluate the myocardium contractility velocity by the technique of TDI in healthy cats submitted to dissociative anesthesia. For that purpose, there were used nine cats, all adult, healthy and without sexual or breeding distinction. There were measured the values of Ea (early diastolic peak velocity), Aa (late diastolic peak velocity) and Sa (systolic peak velocity) in the lateral and septum wall sides. The mean values observed to septum Ea and septum Sa were of $7.2 \pm 2.5 \mathrm{~cm} \mathrm{~s}^{-1}$ and $6.3 \pm 1.7 \mathrm{~cm} \mathrm{~s}^{-1}$, respectively. The mean value obtained for lateral Ea was of $12.0 \pm 4.1 \mathrm{~cm} \mathrm{~s}^{-1}$, while the mean value of lateral Sa was of $8.3 \pm 1.6 \mathrm{~cm} \mathrm{~s}^{-1}$. It was obtained a mean value of $7.0 \pm 3.1 \mathrm{~cm} \mathrm{~s}^{-1}$ for the lateral $A a$, and $4.7 \pm 1.5 \mathrm{~cm} \mathrm{~s}^{-1}$ for septum Aa. The standard of the myocardium contraction velocities mean values in the feline specie is of great importance, and it may help in the early diagnosis of heart contraction alterations. Due to the lack of studies with the TDI technique in cats, the values obtained in our work allow the realization of comparative studies, helping to apply this technique in experimental protocols and to the early diagnosis of cardiomyopathies in the feline specie.
\end{abstract}

Key words: feline, cardiology, diastolic function.

IDepartamento de Medicina Veterinária, Centro de Ciências Agrárias (CCA), Universidade Federal do Espírito Santo (UFES), 29500-000, Alegre, ES, Brasil. E-mail: danielborlini@hotmail.com. *Autor para correspondência.

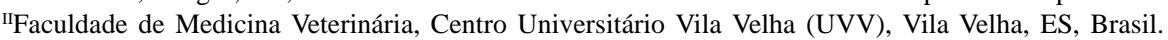

IIIHospital Universitário Cassiano Antônio de Moraes, UFES, Vitória, ES, Brasil. 


\section{INTRODUÇÃO}

Os gatos domésticos podem ser acometidos por diversas cardiopatias, podendo estas ser congênitas ou adquiridas. As afecções do miocárdio são as alterações cardíacas que ocorrem com maior frequência nessa espécie e, em parte dos casos atendidos, a sua causa precisa não é determinada (FOX, 1999). A maioria das miocardiopatias é considerada como primária e idiopática, sendo a miocardiopatia hipertrófica a apresentação clínica mais frequentemente diagnosticada (BONAGURA \& LEHMUKUHL, 1999). Apesar disso, podem ser identificadas formas secundárias de alterações do miocárdio, como, por exemplo, no hipertiroidismo, na hipertensão sistêmica, na acromegalia e na deficiência nutricional de taurina.

Os exames complementares são de grande valor para a investigação do sistema cardiovascular felino, uma vez que, normalmente, é tecnicamente mais difícil realizar uma precisa avaliação clínica em gatos cardiopatas (LORD \& ZONTINE, 1977). O exame ecocardiográfico proporciona um método diagnóstico preciso, simples, não invasivo e que não utiliza radiação ionizante, permitindo a visualização das estruturas internas do coração (PIPERS \& HAMLIN, 1980; DEMADRON et al., 1985; BOND et al., 1988; KIENLE et al., 1997).

Informações sobre o fluxo de sangue nas câmaras cardíacas e seus vasos, tais como sua direção e velocidade, podem ser obtidas pela função Doppler. Uma adequada investigação dos aspectos hemodinâmicos realizada por meio desse exame pode ser determinante para o estabelecimento de um diagnóstico definitivo nos animais cardiopatas (BOON, 1998).

O Doppler tecidual é uma técnica relativamente nova na medicina e que permite avaliar, de forma quantitativa, a velocidade de movimentação do miocárdio, tanto na sístole, como na diástole (NAGUEH et al., 1997; SOHN et al., 1997; ARAÚJO SOBRINHO, 2005; ARINC et al., 2006; DI BELLO et al., 2007). A utilização do Doppler tecidual, em comparação com a ecocardiografia bidimensional, proporciona melhor avaliação da função segmentar do miocárdio, assim como permite uma adequada avaliação da função diastólica do coração (MCDICKEN et al., 1992; SILVA et al., 2002).

O desenvolvimento do Doppler tecidual foi baseado em diferenças físicas existentes entre a movimentação do músculo cardíaco e a do sangue. As velocidades de movimentação do miocárdio e do fluxo sanguíneo diferem substancialmente. Enquanto o miocárdio se movimenta com uma velocidade de 4 a $15 \mathrm{~cm} \mathrm{~s}^{-1}$, a velocidade do fluxo sanguíneo é em torno de 40 a $150 \mathrm{~cm} \mathrm{~s}^{-1}$. O Doppler convencional utiliza um filtro específico que elimina ruídos de baixa frequência e alta amplitude, que são originados da parede ventricular. Com a substituição desse filtro por um que permita a aquisição dos sinais do miocárdio, é possível obter a velocidade de movimentação em qualquer ponto específico do músculo cardíaco (PALKA et al., 1995).

Na medicina veterinária, essa técnica é utilizada apenas para fins científicos e, ainda assim, existem poucos estudos com Doppler tecidual em animais (CHETBOUL et al., 2004; CHETBOUL et al., 2005; CHETBOUL et al., 2006a; CHETBOUL et al., 2006b; CHETBOUL et al., 2006c; KOFFAS et al., 2006; MACDONALD et al., 2006; MACDONALD et al., 2007).

O objetivo deste trabalho foi estabelecer valores médios das velocidades de pico sistólica e diastólica do miocárdio do ventrículo esquerdo, obtidos nas bordas septal e parietal do anel mitral do coração de gatos hígidos por meio da técnica de Doppler tecidual, com a finalidade de obter dados da função contrátil cardíaca para a espécie felina.

\section{MATERIAL E MÉTODOS}

Foram utilizados neste experimento nove gatos domésticos (Felis catus, L. 1758), adultos jovens, sem raça definida, sem distinção de sexo, e com peso

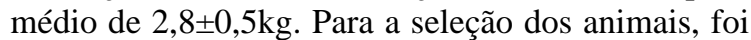
realizada previamente uma avaliação clínica do sistema cardiovascular, realizada por meio de auscultação dos focos cardíacos mitral, tricúspide, pulmonar e aórtico, palpação do pulso femoral echoque de ponta, além de exames eletrocardiográficos e ecoDopplercardiográficos a fim de excluir animais que não se apresentassem dentro dos padrões de normalidade segundo TILLEY \& GOODWIN (2002). Após a seleção, os animais foram alojados em um gatil com acesso ao sol, sendo fornecida alimentação (ração seca) e água à vontade.

Para a realização dos exames ecocardiográficos com Doppler tecidual, foi feita anestesia dissociativa com tiletamina e zolazepam $\left(0,12 \mathrm{ml} \mathrm{kg}^{-1}\right.$ de peso), associados com midazolam $(0,1 \mathrm{mg}$ $\mathrm{kg}^{-1}$ de peso), ambos por via intramuscular. Antes da administração desses fármacos, foi realizado jejum hídrico de 8 horas e alimentar de 12 horas.

Após avaliação ecocardiográfica transtorácica convencional, foram aferidas as velocidades de pico sistólica e diastólica do miocárdio do ventrículo esquerdo pela técnica de Doppler tecidual. Para a obtenção desses valores, foi obtida imagem ecocardiográfica apical quatro câmaras, sendo selecionadas as áreas das bordas lateral e septal do anel mitral (Figura 1). Os exames foram armazenados 


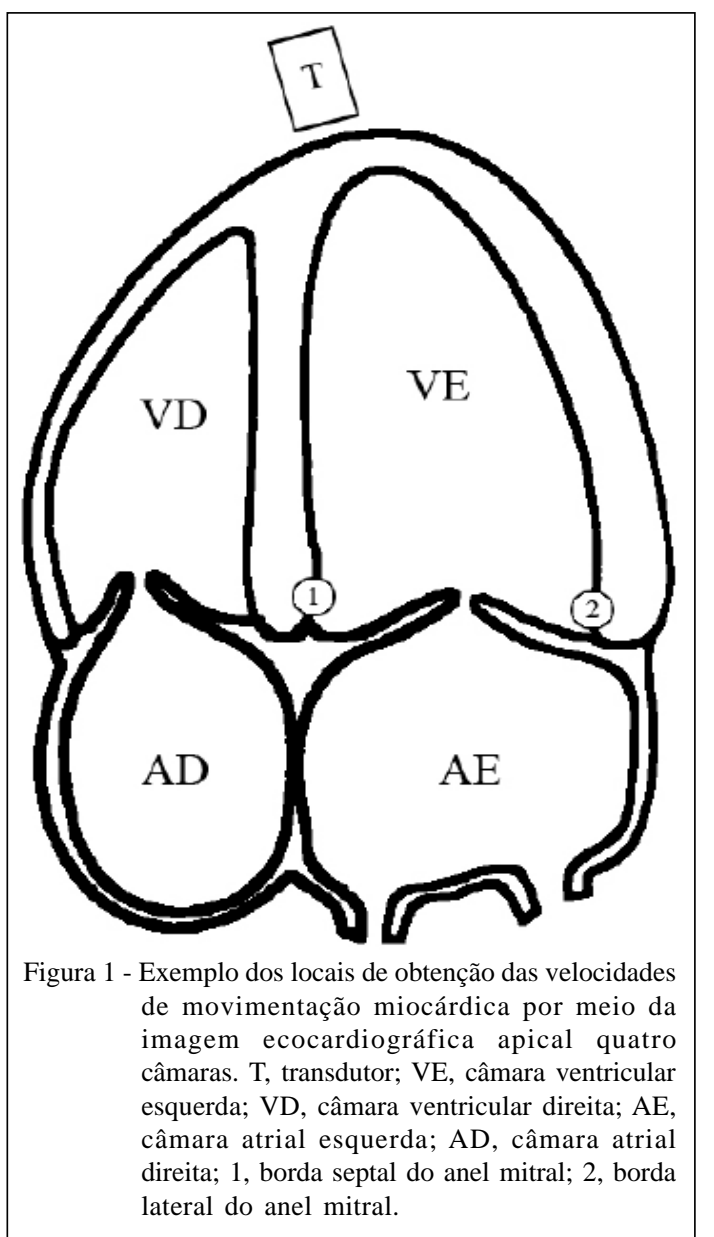

em formato digital para posterior análise e tabulação dos valores das variáveis em cada segmento avaliado.

As medidas de Doppler tecidual utilizadas para a avaliação da função miocárdica de gatos normais foram os valores de Ea (velocidade máxima longitudinal no início da diástole), Aa (velocidade máxima longitudinal na sístole atrial) e Sa (velocidade máxima longitudinal na sístole ventricular) nas bordas lateral e septal do anel mitral (Figura 2). Posteriormente, os dados foram submetidos à análise estatística descritiva, sendo calculadas as médias e os desvios-padrão.

\section{RESULTADOS E DISCUSSÃO}

Conforme demonstrado na tabela 1 , os valores médios observados para Ea septal e Sa septal foram de $7,2 \pm 2,5 \mathrm{~cm} \mathrm{~s}^{-1}$ ede $6,3 \pm 1,7 \mathrm{~cm} \mathrm{~s}^{-1}$, respectivamente. O valor médio obtido para Ea lateral foi de $12,0 \pm 4,1 \mathrm{~cm} \mathrm{~s}^{-1}$, enquanto que o valor médio de Sa lateral foi de $8,3 \pm 1,60 \mathrm{~cm} \mathrm{~s}^{-1}$. Foi obtido um valor médio de $7,0 \pm 3,1 \mathrm{~cm} \mathrm{~s}^{-1}$ para Aa lateral e $4,7 \pm 1,5 \mathrm{~cm} \mathrm{~s}^{-1}$ para Aa septal.
Grande parte dos estudos na espécie felina são direcionados para animais com hipertrofia do miocárdio ventricular (CHETBOUL et al., 2006a; CHETBOUL et al., 2006b; KOFFAS et al., 2006; MACDONALD et al., 2006; MACDONALD et al., 2007). Porém, parâmetros de normalidade são pouco abordados na literatura (CHETBOUL et al., 2006c; KOFFAS et al., 2006).

KOFFAS et al. (2006) realizaram avaliação cardiovascular pela técnica de Doppler tecidual em 25 gatos normais, sendo 21 da raça pelo curto americano, sem realização de contenção química, obtendo resultados próximos aos descritos neste artigo (Tabela 1). CHETBOUL et al. (2006c) também realizaram estudos em 100 gatos normais não anestesiados; entretanto, os pontos de análise foram diferentes dos pontos realizados na presente pesquisa, sendo avaliada a movimentação de outras porções do miocárdio ventricular, impossibilitando, dessa forma, uma comparação entre os valores das variáveis mensuradas em cada trabalho.

Deve-se levar em consideração alguns fatores para efeito comparativo, uma vez que, segundo CHETBOUL et al. (2006c), foi detectado um efeito racial sobre todas as variáveis do Doppler tecidual em uma população de 100 gatos normais. Gatos de raças grandes, como o Maine Coon, tendem a ter valores mais elevados em todas as variáveis mensuradas pelo Doppler tecidual, enquanto raças de porte inferior, como o pelo curto americano, têm valores menores.

A proximidade dos valores obtidos neste estudo com aqueles obtidos por KOFFAS et al. (2006) demonstram que o protocolo anestésico pouco influenciou as mensurações realizadas pela técnica de Doppler tecidual, não sendo observado um déficit na função diastólica dos animais. Esse achado concorda com os relatos de HELLYER et al. (1988), os quais citam que os efeitos cardiovasculares decorrentes da realização de anestesia dissociativa com tiletamina e zolazepam em gatos se normalizam após um minuto de sua aplicação.

Resultados de ecocardiografias e estudos de necropsia indicam que as cardiomiopatias são a mais séria categoria de doenças cardíacas em gatos. O diagnóstico precoce das cardiomiopatias é uma grande preocupação porque essas doenças são as causas mais frequentes de morte súbita, insuficiência cardíaca congestiva e tromboembolismo em gatos (CHETBOUL, 2006a). Dessa forma, a técnica de Doppler tecidual apresenta grande importância para antecipar a identificação de alterações do miocárdio ventricular e auxiliar na prevenção de complicações secundárias, pois se apresenta como um exame de maior sensibilidade 


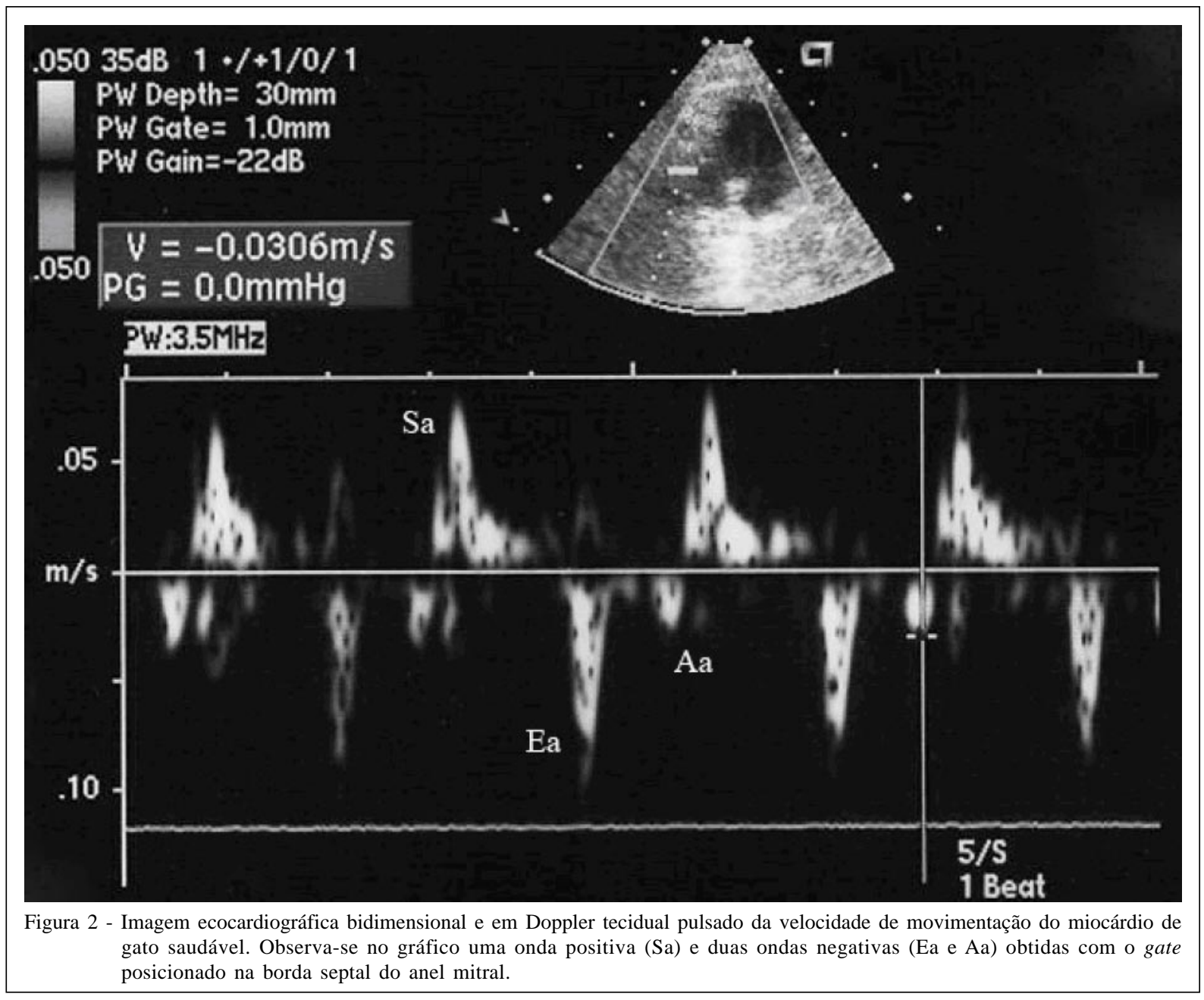

em comparação à ecoDopplercardiografia convencional, sendo capaz de indicar mais precocemente distúrbios na função contrátil do músculo cardíaco.

O Doppler tecidual tem sido usado no diagnóstico de disfunção diastólica em humanos e em animais com vários tipos de doenças cardíacas, incluindo cardiomiopatia hipertrófica, fibrose endomiocárdica, doenças hipertensivas do coração, cardiomiopatia restritiva, doença isquêmica e cardiomiopatia dilatada. As medidas de Ea discriminam com precisão (87\% de sensibilidade e $97 \%$ de especificidade) pacientes com cardiomiopatia hipertrófica de atletas com hipertrofia fisiológica e de pacientes com doença hipertensiva cardíaca (MACDONALD et al., 2006). Assim como em humanos, neste trabalho foi verificado que essa técnica possui grande aplicabilidade para melhor avaliar o sistema cardiovascular felino, podendo também contribuir para o diagnóstico e monitoramento de diversas cardiomiopatias.
Segundo SOHN et al. (1997) e NAGUEH et al. (1997), o Doppler tecidual tem se mostrado um excelente método em humanos para a análise não invasiva da função diastólica, pois é menos sensível à interferência da pré-carga que a mensuração do influxo mitral pelo Doppler tradicional e por isso seus resultados são mais precisos e de maior auxílio na identificação de alterações cardiovasculares. Essa vantagem também deve ser considerada para a espécie felina, aumentando a precisão da avaliação diastólica.

Conforme citado por LORD \& ZONTINE (1977), os gatos cardiopatas apresentam maior dificuldade para a realização de uma precisa avaliação clínica, portanto a utilização do Doppler tecidual, em conjunto com outros métodos diagnósticos, é mais uma ferramenta de grande importância para a investigação do sistema cardiovascular felino.

\section{CONCLUSÕES}

O exame ecocardiográfico com Doppler tecidual na prática clínica propiciará uma nova 
Tabela 1 - Valores médios obtidos pela técnica de Doppler tecidual em gatos normais. Dados representados como média \pm desvio padrão e comparados com os valores encontrados por KOFFAS et al. (2006).

\begin{tabular}{lcc}
\hline & $\begin{array}{c}\text { Média } \pm \text { Desvio } \\
\text { Padrão }\end{array}$ & $\begin{array}{c}\text { KOFFAS et al. } \\
(2006)\end{array}$ \\
\hline Ea lateral $\left(\mathrm{cm} \mathrm{s}^{-1}\right)$ & $12,0 \pm 4,1$ & $8,4 \pm 2,6$ \\
Ea septal $\left(\mathrm{cm} \mathrm{s}^{-1}\right)$ & $7,2 \pm 2,5$ & $6,4 \pm 1,8$ \\
Sa lateral $\left(\mathrm{cm} \mathrm{s}^{-1}\right)$ & $8,3 \pm 1,6$ & $7,1 \pm 2,3$ \\
Sa septal $\left(\mathrm{cm} \mathrm{s}^{-1}\right)$ & $6,3 \pm 1,7$ & $7,0 \pm 1,8$ \\
Aa lateral $\left(\mathrm{cm} \mathrm{s}^{-1}\right)$ & $7,0 \pm 3,1$ & $6,2 \pm 2,4$ \\
Aa septal $\left(\mathrm{cm} \mathrm{s}^{-1}\right)$ & $4,7 \pm 1,5$ & $6,6 \pm 2,4$ \\
\hline
\end{tabular}

Sa lateral, velocidade máxima longitudinal da borda lateral do anel mitral na sístole ventricular; Sa septal, velocidade máxima longitudinal da borda septal do anel mitral na sístole ventricular; Ea lateral, velocidade máxima longitudinal da borda lateral do anel mitral no início da diástole ventricular; Ea septal, velocidade máxima longitudinal da borda septal do anel mitral no início da diástole ventricular; Aa lateral, velocidade máxima longitudinal da borda lateral do anel mitral na sístole atrial; Aa septal, velocidade máxima longitudinal da borda septal do anel mitral na sístole atrial.

ferramenta diagnóstica até então não muito utilizada na medicina veterinária, fornecendo importantes informações sobre os aspectos fisiológicos ou fisiopatológicos em diferentes enfermidades.

As variáveis mensuradas no presente trabalho se aproximam de valores já descritos para gatos não anestesiados, demonstrando pouca interferência da anestesia dissociativa na função diastólica de gatos normais. Esses valores poderão ser utilizados como base em estudos similares, proporcionando futuramente diagnósticos precisos e de qualidade, utilizando a técnica do Doppler tecidual na avaliação do sistema cardiovascular de pequenos animais.

\section{COMITÊ DE ÉTICA E BIOSSEGURANÇA}

Este estudo foi realizado sob aprovação do Comitê de Ética e Bem-estar Animal da Universidade Estadual Paulista, Campus de Botucatu, sob protocolo 49/2002-CEEA, tendo sido respeitados todos os preceitos éticos de proteção aos animais.

\section{REFERÊNCIAS}

ARAÚJO SOBRINHO, A.Q. Efeito do antagonismo de angiotensina II em pacientes portadores de cardiomiopatia hipertrófica não obstrutiva. 2005. 85f. Tese (Doutorado em Ciências) - Faculdade de Medicina da Universidade de São Paulo, São Paulo. Disponível em: <http://www.teses.usp.br/teses/ disponiveis/5/5131/tde-02042007-104427/>. Acesso em: 02 jun. 2009.

ARINC, H. et al. Evaluation of right ventricular function in patients with thyroid dysfunction. Cardiology, v.105, n.2, p.89-94, 2006.
BONAGURA, J.D.; LEHMKUHL, L.B. Congenital heart disease. In: FOX, P.R.et al. Textbook of canine and feline cardiology. Philadelphia: Saunders, 1999. p.471-535.

BOND, B.R. et al. Echocardiographic findings in 103 cats with hyperthyroidism. Journal of American Veterinary Medical Association, v.192, n.11, p.1546-1549, 1988.

BOON, J.A. Manual of veterinary echocardiography. Baltimore: Lippincott Williams \& Wilkins, 1998. 478p.

CHETBOUL, V. et al. Tissue Doppler imaging detects early asymptomatic myocardial abnormalities in a dog model of Duchenne's cardiomyopathy. European Heart Journal, v.25, p.1934-1939, 2004. Disponível em: <http://eurheartj.oxfordjournals.org/cgi/content/ abstract/25/21/1934>. Acesso em: 02 jun. 2009. doi:10.1016/ j.ehj.2004.09.007.

CHETBOUL, V. et al. Use of quantitative two-dimensional color tissue Doppler Imaging for assessment of left ventricular radial and longitudinal myocardial velocities in dogs. American Journal of Veterinary Research, v.66, n.6, p.953-961, 2005.

CHETBOUL, V. et al. Tissue Doppler imaging for detection of radial and longitudinal myocardial dysfunction in a family of cats affected by dystrophin-deficient hypertrophicmuscular dystrophy. Journal of Veteterinary Internal Medicine, v.20, p.640-647, 2006a.

CHETBOUL, V. et al. Two-dimensional color tissue Doppler Imaging detects myocardial dysfunction before occurrence of hypertrophy in a young Maine Coon cat. Veterinary Radiology \& Ultrasound, v.47, n.3, p.295-300, 2006b. Disponível em: <http://www3.interscience.wiley.com/cgi-bin/ fulltext/118574960/PDFSTART>. Acesso em: 02 jun. 2009. doi: 10.1111/j.1740-8261.2006.00143.x.

CHETBOUL, V. et al. Quantitative assessment of velocities of the annulus of the left atrioventricular valve and left ventricular free wall in healthy cats by use of two-dimensional color tissue Doppler imaging. American Journal of Veterinary Research, v.67, n.2, p.295-300, 2006c.

DEMADRON, E. et al. Two-dimensional echocardiography in the normal cat. Veterinary Radiology \& Ultrasound, v.26, n.5, p.149-158, 1985.

DI BELLO, V. et al. Abnormalities of left ventricular myocardial characteristics associated with subclinical hyperthyroidism. Journal of Endocrinology Investigation, v.30, n.7, p.564571, 2007.

FOX, P.R. Feline cardiomyopathies. In: FOX P.R. et al. Textbook of canine and feline cardiology, principles and clinical practice. Philadelphia: Saunders, 1999. p.621-678.

HELLYER, P. et al. Cardiorespiratory effects of the intravenous administration of tiletamine-zolazepam to cats. Veterinary Surgery, v.17, n.2, p.105-109, 1988.

KIENLE R.D. et al. Biplane transesophageal echocardiography in the normal cat. Veterinary Radiology \& Ultrasound. v.38, n.4, p.288298, 1997. Disponível em: <http://www3.interscience.wiley.com/cgibin/fulltext/119162138/PDFSTART>. Acesso em: 02 jun. 2009. doi: 10.1111/j.1740-8261.1997.tb00858.x. 
KOFFAS, H. et al. Pulsed Tissue Doppler imaging in normal cats and cats with hypertrophic cardiomyopathy. Journal of Veterinary Internal Medicine, v.20, p.65-77, 2006. Disponível em: <http://www3.interscience.wiley.com/cgi-bin/ fulltext/119820306/PDFSTART>. Acesso em: 02 jun. 2009. doi: 10.1111/j.1939-1676.2006.tb02825.x.

LORD P.F.; ZONTINE W.J. Radiologic examination of the feline cardiovascular system. Veterinary Clinics of North America, v.7, p.291-308, 1977.

MACDONALD, K.A. et al. Tissue Doppler imaging and gradient echo cardiac magnetic resonance imaging in normal cats and cats with hypertrophic cardiomyopathy. Journal of Veterinary Internal Medicine, v.20, p.627-634, 2006. Disponível em: <http://www3.interscience.wiley.com/cgi-bin/ fulltext/119820335/PDFSTART>. Acesso em: 02 jun. 2009. doi: 10.1111/j.1939-1676.2006.tb02907.x.

MACDONALD, K.A. et al. Tissue Doppler imaging in maine coon cats with a mutation of myosin binding protein $C$ with or without hypertrophy. Journal of Veterinary Internal Medicine, v. 21, n.2, p.232-237, 2007. Disponível em: <http:/ /www3.interscience.wiley.com/cgi-bin/fulltext/120715427/ PDFSTART>. Acesso em: 02 jun. 2009. doi: 10.1111/j.19391676.2007.tb02954.x

MCDICKEN, W.N. et al. Color Doppler velocity imaging of the myocardium. Ultrasound in Medicine and Biology, v.18, p.651-654, 1992.

NAGUEH, S.F. et al. Doppler tissue imaging: A noninvasive technique for evaluation of left ventricular relaxation and estimation of filling pressures. Journal of the American College of Cardiology, v.30, p.1527-1533, 1997. Disponível em: <http:// www.sciencedirect.com/science?_ob=ArticleURL\&_udi=B6T183VBSR4P-M\&_user $=686358 \&$ \& rdoc $=1 \&$ \& fmt $=\&$ \& or $\mathrm{g}=\mathrm{s}$ e a r c h \&_s or t $=$ d \& vi e w $=$ c \&_a c c t $=$ C 0 $00037498 \&$ version $=1 \&$ urlversion =0\&_userid=686358\&md5=5642198e66b38af955cd54ead2f50bdb $>$. Acesso em: 02 jun. 2009. doi: 10.1016/S0735-1097(97)00344-6.

PALKA, P. et al. Doppler tissue imaging: myocardial wall motion velocities in normal subjects. Journal of the American Society of Echocardiography, v.8, p.659-658, 1995. Disponível em: < h t t p : / / w w w. s c i e n c e d i r e c t . c o m/ science?_ob=MImg\&_imagekey=B6WMB-4JGG77V-D-

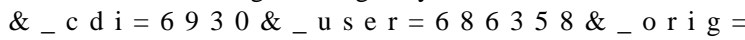
s e a c h \&_c o ver D a t e = $10 \% 2$ F $31 \% 2$ F 1 995\&_sk=999919994.7998\&view $=c \& w c h p=d G L b V I W-$ zSkWA\&md5=0070d78efc3a77dcc6ccd262c7893b4d\&ie=/ sdarticle.pdf $>$. Acesso em: 02 jun. 2009. doi: 10.1016/S08947317(05)80380-3.

PIPERS, F.S.; HAMLIN, R.L. Clinical use of echocardiography in the domestic cat. Journal of American Veterinary Medical Association, v.146, p.57-61, 1980.

SILVA, C.E.S. et al. Estudo das velocidades de contração e relaxamento do miocárdio pela ecocardiografia com Doppler tecidual. Nova alternativa para a avaliação da função ventricular segmentar. Arquivos Brasileiros de Cardiologia, v.78, n.2, p.200-205, 2002. Disponível em: <http://www.scielo.br/pdf/ \%0D/abc/v78n2/p09v78n2.pdf>. Acesso em: 02 jun. 2009. doi: 10.1590/S0066-782X2002000200009.

SOHN, D.W. et al. Assessment of mitral annulus velocity by Doppler tissue imaging in the evaluation of left ventricular diastolic function. Journal of the American College Cardiology, v.30, p.474-480, 1997. Disponível em: <http:// www.sciencedirect.com/science?_ob=MImg\&_imagekey=B6T183SHT4PW-3K-H\&_cdi $=4884 \&$ \&user $=686358$ \&_orig $=$ searc h \&_c o v e r D a t e = $\begin{array}{llllllllllll}0 & 8 & 2 & \text { F } 3 & 1 & \% & 2 & \mathrm{~F} & 1 & 9 & 9 & 7\end{array}$ \&_s k $=999699997$ \& vi e w $=$ c \& w c h p $=$ d G L z V l z zSkzS\&md5=7b37bb49ebde426ca4ae2966634a2d2a\&ie=/ sdarticle.pdf >. Acesso em: 02 jun. 2009. doi: 10.1016/S07351097(97)88335-0 